\title{
Virtual Reality Authoring Tools for Educators
}

\author{
Silviu Vert ${ }^{1, *}$, Diana Andone ${ }^{1}$ \\ ${ }^{1}$ Politehnica University of Timisoara, Multimedia Center, Bd. Vasile Pârvan Nr. 2, 300223, \\ Timisoara, Romania
}

\begin{abstract}
Virtual reality technologies have been demonstrated to produce encouraging results in the educational process. However, adoption of virtual reality-based instruction among educators has yet to see significant numbers. One of the reasons is the technical difficulty in authoring custommade VR experiences for students. In this paper, we propose criteria for choosing VR authoring tools that are appropriate for usage in education and apply them to such current platforms. We also present a case study of learning collaboration project that revolved around the usage of VR authoring tools. We conclude with some recommendations regarding additional features that would enhance the exploitation of current VR authoring tools in education.
\end{abstract}

\section{Introduction}

Virtual reality (VR) technologies are being used in education to create engaging and memorable learning experiences for the students.

There are many examples of VR usage and usefulness in education, such as in the fields of geography, history, engineering, biology and others [1]-[4].

While educators seem to be aware of the potential of these new technologies, there is a barrier to their adoption in mainstream education. This paper focuses on the accessibility from a technical point of view - of authoring tools that allow teachers to design their own custom-made VR experience for use in class.

While pre-made VR applications have popped up in various areas of education, teachers need mostly to develop their own custom VR experience. There have been attempts from the academia to propose and implement VR authoring tools, while the commercial sector is also working hard in this area, with several well-positioned products.

We look at the current offerings and filter the VR authoring tools based on criteria that makes them appropriate for use in education.

To the best of our knowledge, there is no research to compare and asses the usefulness of VR authoring tools for educators, probably because such authoring tools have been developed greatly only in the most recent years.

When we refer to educators in this paper, we also refer to students using directly these authoring tools to design VR experiences and enhance their learning, as we will see in the case study of the TalkTech project. For simplicity, we will only refer to educators.

\footnotetext{
*Corresponding author: silviu.vert@,cm.upt.ro
} 
The paper concludes with recommendations regarding additional features that would greatly enhance the current VR authoring tools for use in education.

\section{Benefits and Adoption of Virtual Reality in Education}

One of the first meta-studies (1998) on the educational uses of virtual reality technology [5] showed that students using VR technology usually performed better (but at least similarly) than those using other forms of instruction, and that students enjoy using VR and are highly motivated in this activity. What was interesting was the effectiveness of student developed VR-environments alongside the usage of pre-developed VR-applications.

A decade and a half later, in 2014, a meta-analysis study provided evidence that Virtual Reality can enhance learning outcomes [6]. The paper analyses studies in the area of virtual reality-based instruction, in K-12 or higher education settings, on games, simulations and virtual worlds, until 2011. Games showed higher learning gains than the other two categories, a fact which is not surprising.

From a constructivist point of view, a Virtual Reality educational environment can provide the students with an active role in their learning; can provide experiential and casebased learning, but also social interaction. [7].

Lately, more and more benefits have been observed in experiments with particular educational VR applications. They can be summarized as follows below [8]:

First of all, visual learning is always more engaging and efficient than written texts. In addition, 3D visual representation is always better for the understanding of complex information than $2 \mathrm{D}$ visuals or text.

Secondly, virtual reality lowers the risk factor, in that students can experiment with VR possibly dangerous situations - from visiting a volcano or doing a risky chemistry experiment - without the actual risk involved. Another important aspect is that, if it is a hands-on activity, the students can repeat it as many times as they wish - an error is not irreversible in VR.

Thirdly, it enables remote access to areas and situations that would otherwise be out of reach. Students can visit distant archaeological places or the International Space Station from the comfort of their classroom.

Fourthly, VR immerses students in real-life situations and is entertaining at the same time, which is a big advantage of this medium on other possibly more mundane types of learning.

According to a GfK survey commissioned by Samsung in 2016, 85\% of teachers agree that VR would have a positive effect on their students, but only $2 \%$ of teachers are using VR content in their classrooms [9]. One of the main reasons is the technical difficulty in designing their own VR lessons.

\section{Virtual Reality Authoring Tools for Educators}

A big challenge for adoption of VR in education is the skills required for educators to design and develop VR-based instruction. Immersive VR in particular requires high programming skills, not to mention that designing a VR-based course is a difficult task for the educator [7]. Because cost of hardware and software is not an insurmountable challenge anymore, the access to intuitive and low skills authoring tool for VR is the next key step for adoption of VR in education.

In this chapter, we review virtual reality authoring tools that are appropriate for usage in education. For our selection, we look specifically to authoring tools that:

- Do not require programming skills, or make them very visual and intuitive; 
- Are under constant maintenance and development, so lessons already created will would not cease to be available in the near future;

- Provide an easily-accessible, web-based platform for creating the experiences (no need to download specific software);

- Can be used for free (even if with limited features);

- And can reach the vast majority of students through wide device/medium distribution (web, Android, iOS, VR headsets or a combination of these).

We apply these criteria to authoring tools that the authors of this paper have searched for on specialized platforms, on Google Scholar or tested in our own experience in teaching fourth-year multimedia undergraduate students and first-year digital communication master students at the Politehnica University of Timisoara.

We arrive at the conclusion that the following authoring tools meet our criteria for successful usage by educators: Cospaces Edu ${ }^{\dagger}$, Uptale Studio*, InstaVR ${ }^{\S}$, WondaVR ${ }^{* *}$, with EON Reality ${ }^{\dagger \dagger}$ and Fieldscapes ${ }^{\$}$ coming close (they require downloading the creator app on your computer).

A detailed comparison between these platforms would quickly become obsolete, as they constantly upgrade features and add new ones in a rapid manner.

To demonstrate how these VR authoring tools work, we resume below how one of them, CoSpaces, handles the creation and distribution of a VR experience.

To design a VR artefact with CoSpaces, one can use a large selection of 3D assets from the platform's library, such as characters, building blocks, graphics and other 3D objects. After placing them in the $3 \mathrm{D}$ space, users can choose to personalize the environment by uploading a 360-degree picture, either downloaded from the web or taken by the users themselves (figure 1). The latter can be easily achieved with a free panoramic camera smartphone application, such as Google Street View, or with a professional camera such as Samsung 360. Stage images and sounds can be added as well. Lastly, the 3D objects can be made interactive by using a visual programming language, namely CoBlocks, which is similar to Scratch in that it does allow the creation of complex effects without writing a line of code (figure 2). More advanced coding can be achieved with Javascript and Typescript.

After designing, developing, and coding their virtual worlds with the CoSpaces web application, users can explore the virtual worlds on their mobile devices using the CoSpaces mobile app, available for Android and iOS devices. The mobile app enables users to visit these virtual worlds using a Google Cardboard headset or similar VR viewers, creating a fully immersive experience (figure 3). Users may share their worlds with others by providing a link or a QR code.

Due to the features described above (virtual reality, interactivity, personalization), the type of experience that CoSpaces offers is vastly more immersive and instructional than what students can achieve by simply exploring a location with Google Maps / Earth / Street View.

\footnotetext{
$\dagger$ https://cospaces.io/

$\$$ https://www.uptale.io/

$\S$ http://www.instavr.co/

** https://www.wondavr.com/

$\dagger$ https://www.eonreality.com/platform/

$\dagger$ https://www.fieldscapesvr.com/
} 


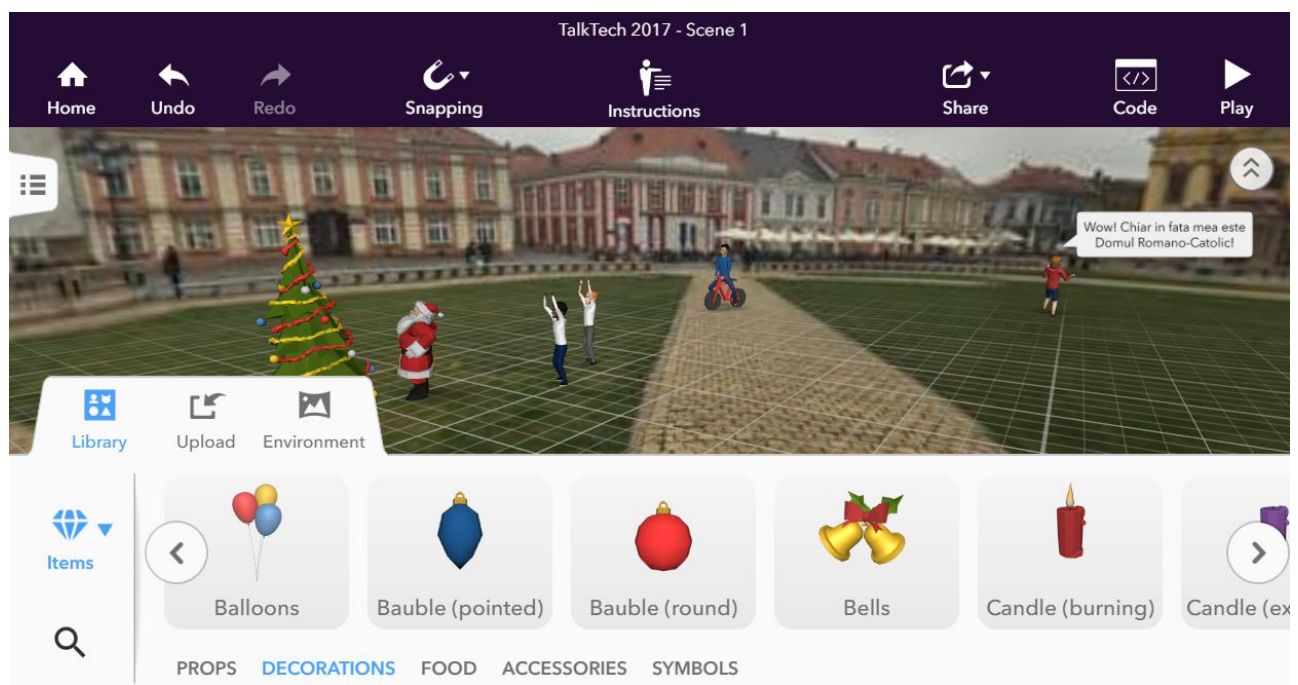

Fig. 1 A virtual world created in CoSpaces, as seen in the editing interface in a browser

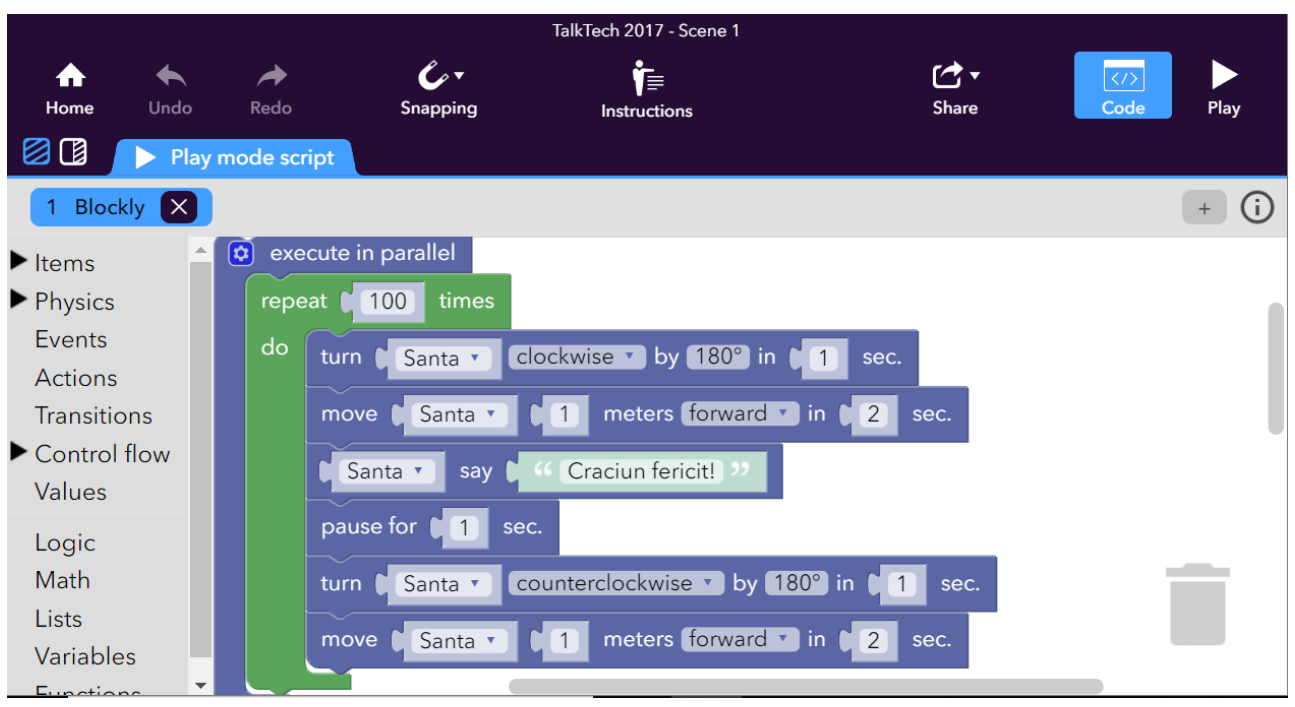

Fig. 2 CoBlocks code to animate the VR artefact 


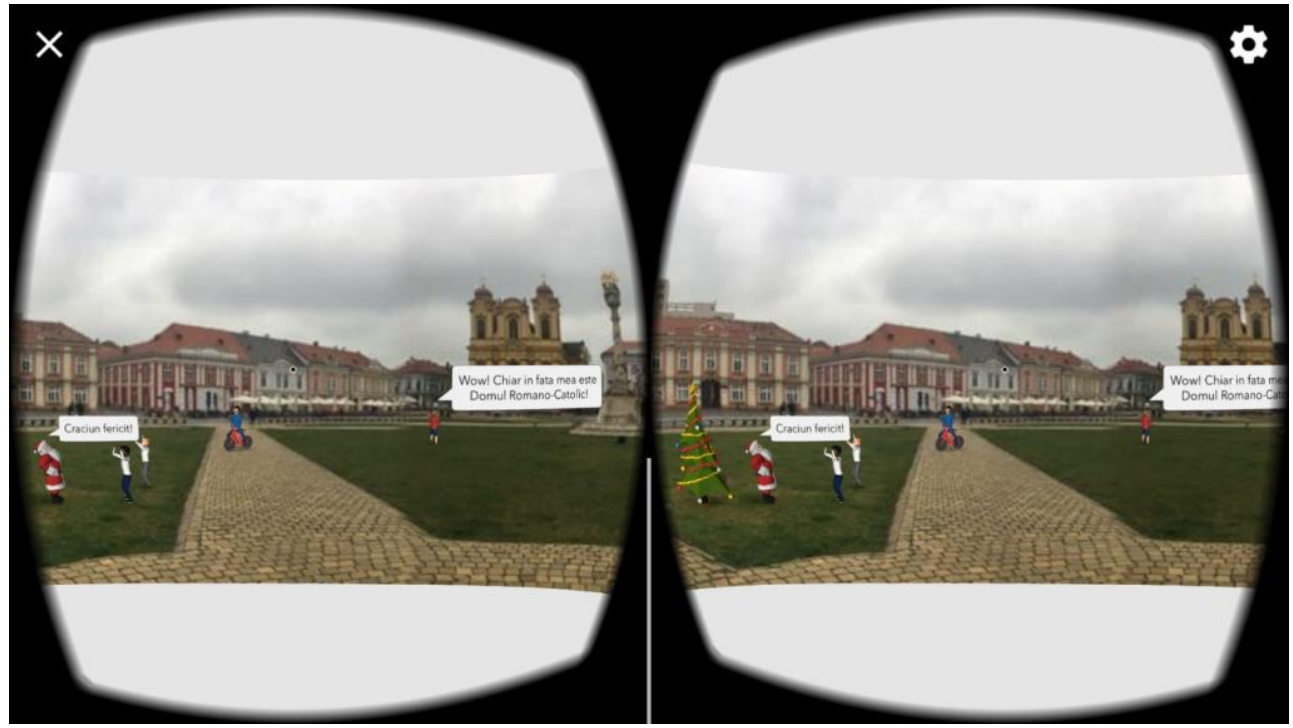

Fig. 3 The same virtual world created in CoSpaces, as seen in a VR viewer

In a nutshell, the typical features that these VR authoring tools offer are:

- Educators can upload their own 360 photos, 360 videos, 2D / 3D models or audio files or can pick from pre-existing ones in the library of the platform.

- Educators can create complex controls and interactions in the VR experience, to build a story for the students.

- Once finished, the VR experience can be played in the browser or in the platform's mobile app, as either immersive (cardboard-style VR) or non-immersive mode.

- Educators and students can share the VR experiences that were created, through links and QR-codes.

- Some analytics are generated by the platform for the educator to study later.

\section{Case study: TalkTech 2017 Project}

TalkTech is a collaborative learning project that, since 2008, has paired students enrolled in a first-year introductory technology course at Bentley University in the United States with fourth-year multimedia students from Politehnica University of Timisoara in Romania, to research, debate current technologies, and create digital media artefacts and experiences that develop computational thinking and digital literacy skills [10], [11].

The TalkTech 2017 project revolved around the VR technology, tools and platforms, as 67 Romanian and American students worked in international teams (2-3 from each country per team) and 46 Romanian students in local teams, to create VR experiences around a cultural or business landmark and share, discuss them with their team partners.

Such locations exploited in VR included cultural landmarks (concert halls, theatres, museums); tech retail shops (Microsoft or Apple store, or other mobile or tech shop); public art (statues, sculptures, building murals); coffee shops, fast food, or local restaurants; sports venues; supermarkets; local landmarks (cathedral, church, park, green, city square); campus landmarks (library, computer lab, classroom); new technology (mobile devices, smart cars, drones). 
The criteria described in the previous chapter is relevant also for student use of VR authoring tools.

Figures 1, 2 and 3 from the previous chapter are an example of student-developed projects, a VR artefact for exploring the Union Square in Timisoara, Romania, during winter. Other student projects included exploring an art gallery, the city's old fortress, a cathedral or the university's library and labs.

Of the 113 students involved in the project, 86 completed a final questionnaire. Although the questionnaire regarded all the aspects of the project (how the project developed, how the students communicated between them, what skills they acquired etc), there were also some questions about the technologies used, mainly VR, but also AR.

The students described CoSpaces (a platform that more than $95 \%$ of them used) as "free and easy to use", "fun and interactive", with "many video tutorials". Overall, these technologies sparked interest and engagement in the students, as they rated AR/VR apps as "clear and understandable", "productive" and "educational". They reported that "learning to use AR/VR is easy" but also that "AR/VR apps can make learning easier". One student concluded that "VR is awesome, [it offers] so much potential".

\section{Discussion and Conclusions}

In order for these VR authoring tools to be even more useful in education, we see the following features as a must-have for the current VR authoring tools (some have already started to implement these):

Collaboration between authors. Whether we are talking about two or more educators designing together a VR experience for a class or about students designing themselves the VR experience as a team, the platform should allow multiple users to edit the experience simultaneously.

Multiuser experience. The social aspect of the learning process would be highly improved if multiple students could "play" simultaneously in the same VR experience and they could interact and modify the scene live.

Assessment. One of the ways to measure the impact of VR-based learning is by assessing the knowledge and skills that the student acquired through the VR experience. As a basic feature, VR authoring tools should allow educators to insert quizzes in the VR setting. This would require the students to be able to authenticate themselves when entering the VR experience.

Organize students in classes. If students are involved in designing the VR experiences, then there is the need for the teacher to be able to assign requirements and oversee an entire class of students, thus making the "add student to a class" a mandatory feature.

Import VR items. There is increasing number of online libraries, one of the best known being SketchFab, that offer users the possibility to download or embed VR items such as 360 photos, 3D models or audio files. VR authoring tools should allow educators to quickly embed such VR items directly from their interface, since most educators are not aware of the existence of all these libraries.

Offline version. Students should have the possibility to download VR experiences, so they could be used in settings that have none or poor connection to the Internet. It is expected that complex VR experiences are bandwidth-heavy.

In this paper, we reviewed the benefits of using VR in education, but also showed that adoption in mainstream education has yet to come, one of the factors being the technical barrier for educators in designing their own VR experience. We proposed criteria for VR authoring tools that are appropriate for education, namely that they do not require advanced programming skills, are under constant maintenance and development, provide an easily- 
accessible, web-based platform for creating the experiences, can be used for free (even if with limited features) and can reach the vast majority of students through wide device/medium distribution. We applied these criteria to such current platforms to make a short useful list.

We also presented our experience in using these VR authoring tools in the TalkTech 2017 project, a collaborative learning endeavour that connects students from Romania with students from the US through innovative technologies. We conclude that current VR authoring tools should add some further features to enhance their usage in education, namely collaboration between authors, multiuser experience, assessment of student activity, organizing students in classes, wide import of VR items and offline version.

\section{References}

[1] H. Kaufmann, D. Schmalstieg, and M. Wagner, "Construct3D: a virtual reality application for mathematics and geometry education," Education and information technologies, vol. 5, no. 4, pp. 263-276, 2000.

[2] M. Lynch, "20 Top Virtual Reality Apps that are Changing Education - The Tech Edvocate," 11-Mar-2017. [Online]. Available: https://www.thetechedvocate.org/20top-virtual-reality-apps-that-are-changing-education/. [Accessed: 20-May-2018]

[3] EON Experience Portal, "EON 3D Lessons." [Online]. Available: http://www.eonexperience.com/3d-lessons/overview.aspx. [Accessed: 15-May-2018]

[4] Delightex GmbH, "CoSpaces Edu - Gallery," CoSpaces Edu: Create virtually anything. [Online]. Available: https://edu.cospaces.io/Universe [Accessed: 22-May2018]

[5] C. Youngblut, "Educational Uses of Virtual Reality Technology.," INSTITUTE FOR DEFENSE ANALYSES ALEXANDRIA VA, 1998.

[6] Z. Merchant, E. T. Goetz, L. Cifuentes, W. Keeney-Kennicutt, and T. J. Davis, "Effectiveness of virtual reality-based instruction on students' learning outcomes in K12 and higher education: A meta-analysis," Computers \& Education, vol. 70, pp. 2940, 2014.

[7] H.-M. Huang, U. Rauch, and S.-S. Liaw, "Investigating learners' attitudes toward virtual reality learning environments: Based on a constructivist approach," Computers \& Education, vol. 55, no. 3, pp. 1171-1182, 2010.

[8] "The Growth of Virtual Reality in eLearning," Origin Learning Blog | eLearning Solutions | LMS | Mobile Learning | Technology. 14-Mar-2018 [Online]. Available: http://blog.originlearning.com/why-virtual-reality-has-a-bright-future-in-the-elearningworld/. [Accessed: 11-May-2018]

[9] "Teachers Ready for Virtual Reality in Education," Samsung Business Insights. 27Jun-2016 [Online]. Available: https://insights.samsung.com/2016/06/27/teachersready-for-virtual-reality-in-education/. [Accessed: 01-May-2018]

[10] D. Andone and M. Frydenberg, "Becoming Creative Creators: Simulating a Global Workplace using Computational Thinking Practices," in EdMedia: World Conference on Educational Media and Technology, 2014, pp. 564-571.

[11] D. Andone and M. Frydenberg, "Experiences in Online Collaborative Learning with Augmented Reality," presented at the The International Scientific Conference eLearning and Software for Education, Timisoara, Romania, 2017, vol. 2, p. 239. 